\title{
The constrained modified KP hierarchy and the generalized Miura transformations
}

\author{
Jiin-Chang Shaw ${ }^{1}$, and Ming-Hsien $\mathrm{Tu}^{2}$ \\ ${ }^{1}$ Department of Applied Mathematics, National Chiao Tung University, \\ Hsinchu, Taiwan, Republic of China, \\ and \\ 2 Department of Physics, National Tsing Hua University, \\ Hsinchu, Taiwan, Republic of China
}

(October 31, 2018)

\begin{abstract}
In this letter, we consider the second Hamiltonian structure of the constrained modified KP hierarchy. After mapping the Lax operator to a pure differential operator the second structure becomes the sum of the second and the third Gelfand-Dickey brackets defined by this differential operator. We simplify this Hamiltonian structure by factorizing the Lax operator into linear terms.
\end{abstract}


Classical $W$-algebras has played an important role in integrable systems [1]. It's Adler map (see, for example, [2]) from which the $W$-algebras can be constructed as Poisson bracket algebras. A typical example is the $W_{n}$ algebra constructed from the second Gelfand-Dickey (GD) structure of the $n$-th Korteweg-de Vries (KdV) hierarchy [3, 1]. Amazingly, under factorization of the KdV-Lax operator, the second Hamiltonian structure is transformed into a much simpler one in an appropriate space of the modified variables. Thus the factorization not only provides a Miura transformation which maps the $n$-th KdV hierarchy to the corresponding modified hierarchies but also gives a free field realization of the $W_{n}$ algebra. This is what we called the Kupershmidt-Wilson (KW) theorem [5.,6]. In general, the above scheme is encoded in the particular form of the Lax operator and its associated Poisson structure. Several integrable systems have been studied based on this scheme, such as the Kadomtsev-Petviashvili (KP) hierarchy and its reductions [7] 13].

In this letter, we will consider a kind of reduction of the KP hierarchy called constrained modified KP (cmKP) hierarchy [14]. Many properties of the cmKP have been studied, such as bi-Hamiltonian structure [14], Bäcklund transformation [15], modification [16], and conformal property [17], etc. However, a clear and conclusive statement about the associated Poisson structure is still lacking. In the following, we will concentrate on this problem. Especially, we will show that there is an interesting property of the second Poisson structure of the cmKP hierarchy under factorization of the Lax operator into linear terms.

The cmKP hierarchy [14 has the Lax operator of the form

$$
K_{n}=\partial^{n}+v_{1} \partial^{n-1}+\cdots+v_{n}+\partial^{-1} v_{n+1}
$$

which satisfies the hierarchy equations

$$
\partial_{k} K_{n}=\left[\left(K_{n}^{k / n}\right)_{\geq 1}, K_{n}\right] .
$$

The second Poisson bracket associated with the Lax operator was obtained by Oevel and Strampp [14] as follows

$$
\{F, G\}=\int \operatorname{res}\left(\frac{\delta F}{\delta K_{n}} \Theta_{2}\left(\frac{\delta G}{\delta K_{n}}\right)\right)
$$

where $F$ and $G$ are functionals of $K_{n}$ and

$$
\begin{aligned}
\Theta_{2}\left(\frac{\delta G}{\delta K_{n}}\right)= & \left(K_{n} \frac{\delta G}{\delta K_{n}}\right)_{+} K_{n}-K_{n}\left(\frac{\delta G}{\delta K_{n}} K_{n}\right)_{+}+\left[K_{n},\left(K_{n} \frac{\delta G}{\delta K_{n}}\right)_{0}\right]+\partial^{-1} \operatorname{res}\left[K_{n}, \frac{\delta G}{\delta K_{n}}\right] K_{n} \\
& +\left[K_{n}, \int^{x}\left(\operatorname{res}\left[K_{n}, \frac{\delta G}{\delta K_{n}}\right]\right)\right]
\end{aligned}
$$

with

$$
\frac{\delta G}{\delta K_{n}} \equiv \frac{\delta G}{\delta v_{n+1}}+\partial^{-1} \frac{\delta G}{\delta v_{n}}+\cdots+\partial^{-n} \frac{\delta G}{\delta v_{1}}
$$

Recently, Liu Q P [16] conjectured that if the Lax operator $K_{n}$ is factorized as

$$
K_{n}=\partial^{-1}\left(\partial-w_{1}\right) \cdots\left(\partial-w_{n+1}\right)
$$


then in terms of $\left\{w_{i}\right\}$ the Poisson structure (3) can be simplified to

$$
\left\{w_{i}(x), w_{j}(y)\right\}=\left(1-\delta_{i j}\right) \delta^{\prime}(x-y) .
$$

where $\delta^{\prime}(x-y) \equiv \partial_{x} \delta(x-y)$. The cases for $n=1$ and $n=2$ have been explicitly demonstrated in [16]. However, to the best of our knowledge, a general proof for all $n$ is still lacking. It is the main purpose of this letter to give an elegant and simple proof for the general case.

To simplify the Hamiltonian structure (4) let us consider the operator

$$
\begin{aligned}
L_{n+1} & \equiv \partial K_{n}=\partial^{n+1}+v_{1} \partial^{n}+\left(v_{2}+v_{1}^{\prime}\right) \partial^{n-1}+\cdots+\left(v_{n+1}+v_{n}^{\prime}\right) \\
& \equiv \partial^{n+1}+u_{1} \partial^{n}+u_{2} \partial^{n-1}+\cdots+u_{n+1}
\end{aligned}
$$

which is a pure differential operator and the variables $\left\{v_{i}\right\}$ and $\left\{u_{i}\right\}$ are related by

$$
\begin{aligned}
v_{1} & =u_{1}, \\
v_{2} & =u_{2}-u_{1}^{\prime}, \\
& \vdots \\
v_{n+1} & =u_{n+1}-u_{n}^{\prime}+\cdots(-1)^{n} u_{1}^{(n)} .
\end{aligned}
$$

Proposition 1 [17]: With respect to the pure differential operator $L_{n+1}$, the second Poisson bracket (3) now becomes

$$
\{F, G\}=\int \operatorname{res}\left(\frac{\delta F}{\delta L_{n+1}} \Omega\left(\frac{\delta G}{\delta L_{n+1}}\right)\right)
$$

where

$\Omega\left(\frac{\delta G}{\delta L_{n+1}}\right)=\left(L_{n+1} \frac{\delta G}{\delta L_{n+1}}\right)_{+} L_{n+1}-L_{n+1}\left(\frac{\delta G}{\delta L_{n+1}} L_{n+1}\right)_{+}+\left[L_{n+1}, \int^{x}\left(\operatorname{res}\left[L_{n+1}, \frac{\delta G}{\delta L_{n+1}}\right]\right)\right]$.

with

$$
\frac{\delta G}{\delta L_{n+1}} \equiv \partial^{-1} \frac{\delta G}{\delta u_{n+1}}+\partial^{-2} \frac{\delta G}{\delta u_{n}}+\cdots+\partial^{-n-1} \frac{\delta G}{\delta u_{1}}
$$

Besides the standard second GD structure, the last piece of (11) is called the third GD bracket which is compatible with the second one [3]. Hence, under the mapping (8), the Hamiltonian structure (4) has been mapped to the sum of the second and the third GD structure defined by the differential operator $L_{n+1}$.

Now we want to show that this Hamiltonian structure can be simplified via the following factorization

$$
L_{n+1}=\left(\partial-w_{1}\right)\left(\partial-w_{2}\right) \cdots\left(\partial-w_{n+1}\right) .
$$

This yields an expression for each $u_{i}$ (and hence $v_{i}$ ) as a differential polynomial in $\left\{w_{i}\right\}$ (the inverse statement is not true). For example 


$$
\begin{aligned}
& u_{1}=-\left(w_{1}+\cdots+w_{n+1}\right) \\
& u_{2}=\sum_{i<j} w_{i} w_{j}-\sum_{i=0}^{n-1}(n-i) w_{n+1-i}^{\prime}
\end{aligned}
$$

etc. The expression (14) is called the Miura transformation.

Proposition 2: Under the factorization (13), the Poisson structure (10) becomes

$$
\{F, G\}=\sum_{i \neq j} \int\left(\frac{\delta F}{\delta w_{i}}\right)\left(\frac{\delta G}{\delta w_{j}}\right)^{\prime}
$$

i.e., the basic building blocks $\left\{w_{i}\right\}$ satisfy (7).

Proof: First, thanks to the KW theorem [5,6] for the second GD structure, the first two terms of the Poisson bracket (10) can be simplified as follows

$$
\{F, G\}_{2}^{G D}=-\sum_{i=1}^{n+1} \int\left(\frac{\delta F}{\delta w_{i}}\right)\left(\frac{\delta G}{\delta w_{i}}\right)^{\prime}
$$

or

$$
\left\{w_{i}(x), w_{j}(y)\right\}_{2}^{G D}=-\delta_{i j} \delta^{\prime}(x-y)
$$

Thus the remaining tasks are to verify

$$
\int \operatorname{res}\left(\frac{\delta F}{\delta L_{n+1}}\left[L_{n+1}, \int^{x} \operatorname{res}\left[L_{n+1}, \frac{\delta G}{\delta L_{n+1}}\right]\right]\right)=\sum_{i, j=1}^{n+1} \int\left(\frac{\delta F}{\delta w_{i}}\right)\left(\frac{\delta G}{\delta w_{j}}\right)^{\prime} .
$$

Let $l_{i} \equiv\left(\partial-w_{i}\right)$, then $L_{n+1}=l_{1} l_{2} \cdots l_{n+1}$ and

$$
\begin{aligned}
\int \operatorname{res}\left(\frac{\delta F}{\delta L_{n+1}} \delta L_{n+1}\right) & =-\int \operatorname{res}\left(\frac{\delta F}{\delta L_{n+1}} \sum_{i=1}^{n+1} l_{1} \cdots l_{i-1} \delta w_{i} l_{i+1} \cdots l_{n+1}\right) \\
& =-\sum_{i=1}^{n+1} \int \operatorname{res}\left(l_{i+1} \cdots l_{n+1} \frac{\delta F}{\delta L_{n+1}} l_{1} \cdots l_{i-1}\right) \delta w_{i} \\
& =\sum_{i=1}^{n+1} \int \frac{\delta F}{\delta w_{i}} \delta w_{i}
\end{aligned}
$$

which implies

Now

$$
\frac{\delta F}{\delta w_{i}}=-r e s\left(l_{i+1} \cdots l_{n+1} \frac{\delta F}{\delta L_{n+1}} l_{1} \cdots l_{i-1}\right)
$$

$$
\begin{aligned}
\left(\sum_{i=1}^{n+1} \frac{\delta F}{\delta w_{i}}\right)^{\prime} & =-\left[\partial, \operatorname{res}\left(\sum_{i=1}^{n+1} l_{i+1} \cdots l_{n+1} \frac{\delta F}{\delta L_{n+1}} l_{1} \cdots l_{i-1}\right)\right] \\
& =-\sum_{i=1}^{n+1} \operatorname{res}\left(\left[\partial, l_{i+1} \cdots l_{n+1} \frac{\delta F}{\delta L_{n+1}} l_{1} \cdots l_{i-1}\right]\right) \\
& =-\sum_{i=1}^{n+1} \operatorname{res}\left(\left[l_{i}, l_{i+1} \cdots l_{n+1} \frac{\delta F}{\delta L_{n+1}} l_{1} \cdots l_{i-1}\right]\right) \\
& =-\operatorname{res}\left[L_{n+1}, \frac{\delta F}{\delta L_{n+1}}\right] .
\end{aligned}
$$


Hence,

$$
\sum_{i=1}^{n+1} \frac{\delta F}{\delta w_{i}}=-\int^{x} \operatorname{res}\left[L_{n+1}, \frac{\delta F}{\delta L_{n+1}}\right] .
$$

Note that we have substituted $l_{i}$ for $\partial$ in the third line because nothing will change.

Therefore,

$$
\begin{aligned}
\sum_{i, j=1}^{n+1} \int\left(\frac{\delta F}{\delta w_{i}}\right)\left(\frac{\delta G}{\delta w_{j}}\right)^{\prime} & =-\int\left(\sum_{i=1}^{n+1} \frac{\delta F}{\delta w_{i}}\right)^{\prime}\left(\sum_{j=1}^{n+1} \frac{\delta G}{\delta w_{j}}\right) \\
& =-\int \operatorname{res}\left(\left[L_{n+1}, \frac{\delta F}{\delta L_{n+1}}\right] \int^{x} \operatorname{res}\left[L_{n+1}, \frac{\delta G}{\delta L_{n+1}}\right]\right) \\
& =\int \operatorname{res}\left(\frac{\delta F}{\delta L_{n+1}}\left[L_{n+1}, \int^{x} \operatorname{res}\left[L_{n+1}, \frac{\delta G}{\delta L_{n+1}}\right]\right]\right) .
\end{aligned}
$$

Proposition 3: If the Hamiltonian $H_{k}$ of the cmKP hierarchy equations $\partial_{k} K_{n}=$ $\left[\left(K_{n}^{k / n}\right)_{\geq 1}, K_{n}\right]=\Theta_{2}\left(\frac{\delta H_{k}}{\delta K_{n}}\right)$ with respect to the second structure is expressed in terms of $\left\{w_{i}\right\}$ by the Miura transformation, then the corresponding modified equations will be

$$
\partial_{k} w_{i}=\sum_{j \neq i}\left(\frac{\delta H_{k}}{\delta w_{j}}\right)^{\prime}
$$

Proof: This is just a corollary of the Proposition 2.

Finally, we would like to provide another interesting property of the Poisson structure (10) although it is less relevant to the present case. In fact, it has been shown [18 that the Poisson structure (10) can be associated to the operator of the form

$$
L=\partial^{N}+u_{1} \partial^{N-1}+\cdots+u_{N}+\sum_{i=1}^{M} \phi_{i} \partial^{-1} \psi_{i} .
$$

Therefore we can discuss the Poisson structure (10) under the factorization of the Lax operator containing inverse linear terms.

Proposition 4: Let $L$ be a pseudo-differential operator of order $n-m$. If $L$ admits the following factorization (generalized Miura transformation)

$$
L=\left(\partial-a_{1}\right) \cdots\left(\partial-a_{n}\right)\left(\partial-b_{1}\right)^{-1} \cdots\left(\partial-b_{m}\right)^{-1}
$$

then the Poisson structure (10) associated with $L$ becomes

$$
\begin{aligned}
& \left\{a_{i}(x), a_{j}(y)\right\}=\left(1-\delta_{i j}\right) \delta^{\prime}(x-y), \\
& \left\{b_{i}(x), b_{j}(y)\right\}=\left(1+\delta_{i j}\right) \delta^{\prime}(x-y), \\
& \left\{a_{i}(x), b_{j}(y)\right\}=\delta^{\prime}(x-y) .
\end{aligned}
$$

Proof: It has been shown 10 13 that the second GD bracket with respects to the factorization (26) are given by

$$
\begin{aligned}
& \left\{a_{i}(x), a_{j}(y)\right\}_{2}^{G D}=-\delta_{i j} \delta^{\prime}(x-y), \\
& \left\{b_{i}(x), b_{j}(y)\right\}_{2}^{G D}=\delta_{i j} \delta^{\prime}(x-y), \\
& \left\{a_{i}(x), b_{j}(y)\right\}_{2}^{G D}=0 .
\end{aligned}
$$


Hence, we only need to treat the third structure and to show that

$$
\int \operatorname{res}\left(\frac{\delta F}{\delta L}\left[L, \int^{x} \operatorname{res}\left[L, \frac{\delta G}{\delta L}\right]\right]\right)=\int\left(\sum_{i=1}^{n} \frac{\delta F}{\delta a_{i}}+\sum_{j=1}^{m} \frac{\delta F}{\delta b_{j}}\right)\left(\sum_{i=1}^{n} \frac{\delta G}{\delta a_{i}}+\sum_{j=1}^{m} \frac{\delta G}{\delta b_{j}}\right)^{\prime} .
$$

Let $A_{i}=\left(\partial-a_{i}\right)$ and $B_{j}=\left(\partial-b_{j}\right)$ then

$$
\begin{aligned}
\delta F= & \int \operatorname{res}\left(\frac{\delta F}{\delta L} \delta L\right) \\
= & \int \operatorname{res}\left(\frac{\delta F}{\delta L} \sum_{i=1}^{n} A_{1} \cdots A_{i-1} \delta A_{i} \cdots A_{n} B_{1}^{-1} \cdots B_{m}^{-1}\right) \\
& +\int \operatorname{res}\left(\frac{\delta F}{\delta L} A_{1} \cdots A_{n} \sum_{j=1}^{m} B_{1}^{-1} \cdots B_{j-1}^{-1} \delta B_{j}^{-1} \cdots B_{m}^{-1}\right) \\
\equiv & \int\left(\sum_{i=1}^{n} \frac{\delta F}{\delta a_{i}} \delta a_{i}+\sum_{j=1}^{m} \frac{\delta F}{\delta b_{j}} \delta b_{j}\right) .
\end{aligned}
$$

Substituting $\delta A_{i}=-\delta a_{i}$ and $\delta B_{j}^{-1}=B_{j}^{-1} \delta b_{j} B_{j}^{-1}$ into (30) and comparing with (31), we obtain

$$
\begin{aligned}
& \frac{\delta F}{\delta a_{i}}=-\operatorname{res}\left(A_{i+1} \cdots A_{n} B_{1}^{-1} \cdots B_{m}^{-1} \frac{\delta F}{\delta L} A_{1} \cdots A_{i-1}\right) \\
& \frac{\delta F}{\delta b_{j}}=\operatorname{res}\left(B_{j}^{-1} \cdots B_{m}^{-1} \frac{\delta F}{\delta L} A_{1} \cdots A_{n} B_{1}^{-1} \cdots B_{j}^{-1}\right) .
\end{aligned}
$$

Thus

$$
\begin{aligned}
\sum_{i=1}^{n}\left(\frac{\delta F}{\delta a_{i}}\right)^{\prime}+\sum_{j=1}^{m}\left(\frac{\delta F}{\delta b_{j}}\right)^{\prime}= & \sum_{i=1}^{n}\left[\partial, \frac{\delta F}{\delta a_{i}}\right]+\sum_{j=1}^{m}\left[\partial, \frac{\delta F}{\delta b_{j}}\right] \\
= & -\sum_{i=1}^{n} \operatorname{res}\left[A_{i}, A_{i+1} \cdots A_{n} B_{1}^{-1} \cdots B_{m}^{-1} \frac{\delta F}{\delta L} A_{1} \cdots A_{i-1}\right] \\
& +\sum_{j=1}^{m} \operatorname{res}\left[B_{j}, B_{j}^{-1} \cdots B_{m}^{-1} \frac{\delta F}{\delta L} A_{1} \cdots A_{n} B_{1}^{-1} \cdots B_{j}^{-1}\right] \\
= & -\operatorname{res}\left[L, \frac{\delta F}{\delta L}\right]
\end{aligned}
$$

which implies

$$
\sum_{i=1}^{n} \frac{\delta F}{\delta a_{i}}+\sum_{j=1}^{m} \frac{\delta F}{\delta b_{j}}=-\int^{x} \operatorname{res}\left[L, \frac{\delta F}{\delta L}\right]
$$

Now

$$
\begin{aligned}
\int\left(\sum_{i=1}^{n} \frac{\delta F}{\delta a_{i}}+\sum_{j=1}^{m} \frac{\delta F}{\delta b_{j}}\right)\left(\sum_{i=1}^{n} \frac{\delta G}{\delta a_{i}}+\sum_{j=1}^{m} \frac{\delta G}{\delta b_{j}}\right)^{\prime} & =-\int\left(\sum_{i=1}^{n} \frac{\delta F}{\delta a_{i}}+\sum_{j=1}^{m} \frac{\delta F}{\delta b_{j}}\right)^{\prime}\left(\sum_{i=1}^{n} \frac{\delta G}{\delta a_{i}}+\sum_{j=1}^{m} \frac{\delta G}{\delta b_{j}}\right) \\
& =-\int \operatorname{res}\left(\left[L, \frac{\delta F}{\delta L}\right] \int^{x} \operatorname{res}\left[L, \frac{\delta G}{\delta L}\right]\right) \\
& =\int \operatorname{res}\left(\frac{\delta F}{\delta L}\left[L, \int^{x} \operatorname{res}\left[L, \frac{\delta G}{\delta L}\right]\right]\right) .
\end{aligned}
$$


In summary, we have shown that the second Hamiltonian structure of the cmKP hierarchy has a very simple realization. In terms of the variables $\left\{w_{i}\right\}$, the Lax operator $K_{n}$ can be factorized as (6) and the Poisson structure (3) is mapped into a much simpler form (7). We also discuss the Poisson structure (10) under factorization of the Lax operator containing inverse linear terms. The resulting brackets (27) turns out to be simple as well. We hope that we can explore the usage of these brackets in the future.

Note added: after submitting this manuscript we note that similar results are also obtained by Q. P. Liu [19]

Acknowledgments We would like to thank Professor W-J Huang for inspiring discussions and Dr. M-C Chang for reading the manuscript. This work was supported by the National Science Council of the Republic of China under grant No. NSC-86-2112-M-007-020. 


\section{REFERENCES}

$2 \quad$ email address: mhtu@phys.nthu.edu.tw

[1] Bouwknegt P and Schoutens K (ed) 1995 W-symmetry (Singapore: World Scientific)

[2] Dickey L A 1991 Soliton Equations and Hamiltonian Systems (Singapore: World Scientific)

[3] Di Francesco P, Itzykson C and Zuber J B 1991 Commun. Math. Phys. 140543

[4] Dickey L A 1993 Lecture on classical W-algebras (unpublished)

[5] Kupershmidt B A and Wilson G 1981 Invent. Math. 62403

[6] Dickey L A 1983 Commun. Math. Phys. 87127

[7] Cheng Y 1995 Commun. Math. Phys. 171661

[8] Bonora L and Xiong C S 1994 J. Math. Phys. 355781

[9] Bonora L, Liu Q P and Xiong C S 1996 Commun. Math. Phys. 175177

[10] Dickey L A 1995 Lett. Math. Phys. 35229

[11] Yu F 1993 Lett. Math. Phys. 29175

[12] Aratyn H, Nissimov E and Pacheva S 1993 Phys. Lett. 314B 41

[13] Mas J and Ramos E 1995 Phys. Lett. 351B 194

[14] Oevel W and Strampp W 1993 Commun. Math. Phys. 15751

[15] Shaw J C and Tu M H 1996 Miura and auto-Bäcklund transformations for the cKP and cmKP hierarchies (preprint)

[16] Liu Q P 1995 Inv. Prob. 199511205

[17] Huang W J, Shaw J C and Yen H C 1995 J. Math. Phys. 362959

[18] Tu M H 1997 "The nonstandard constrained KP hierarchy and the generalized Miura transformation" (preprint).

[19] Liu Q P 1997 "The Constrained MKP Hierarchy and the Generalized KupershmidtWilson Theorem" (preprint) solv-int/9707012. 